Supporting information for

\title{
Electroreduction of Carbon Dioxide into Selective Hydrocarbons at Low Overpotential Using Isomorphic Atomic Substitution in Copper Oxide
}

Subramanian Nellaiappan, ${ }^{\dagger}$ Ritesh Kumar, ${ }^{\ddagger}$ C. Shivakumara ${ }^{\S}$ Silvia Irusta,,$~ J o r d a n$ A. Hachtel, ${ }^{\perp}$ Juan-Carlos Idrobo, ${ }^{\perp}$ Abhishek K. Singh, ${ }^{\ddagger}$ Chandra Sekhar Tiwary, ${ }^{\#}$ and Sudhanshu Sharma ${ }^{*} \dagger$

†Department of Chemistry, Indian Institute of Technology Gandhinagar, Palaj, Gandhinagar 382355, Gujarat, India

${ }^{\ddagger}$ Materials Research Center and §Solid State and Structural Chemistry Unit, Indian Institute of Science, Bangalore 560012, India

"Department of Chemical Engineering, Nanoscience Institute of Aragon (INA), University of Zaragoza, Zaragoza 50018, Spain

${ }^{\perp}$ Center for Nanophase Materials Sciences, Oak Ridge National Laboratory, Oak Ridge, Tennessee 37830, United States

\#Metallurgical and Materials Engineering, Indian Institute of Technology, Kharagpur 600036, India

*E-mail: ssharma@iitgn.ac.in

Number of pages $\quad: 21$

Number of Figures : : 16

Number of Tables : :06 


\section{Figure S1}
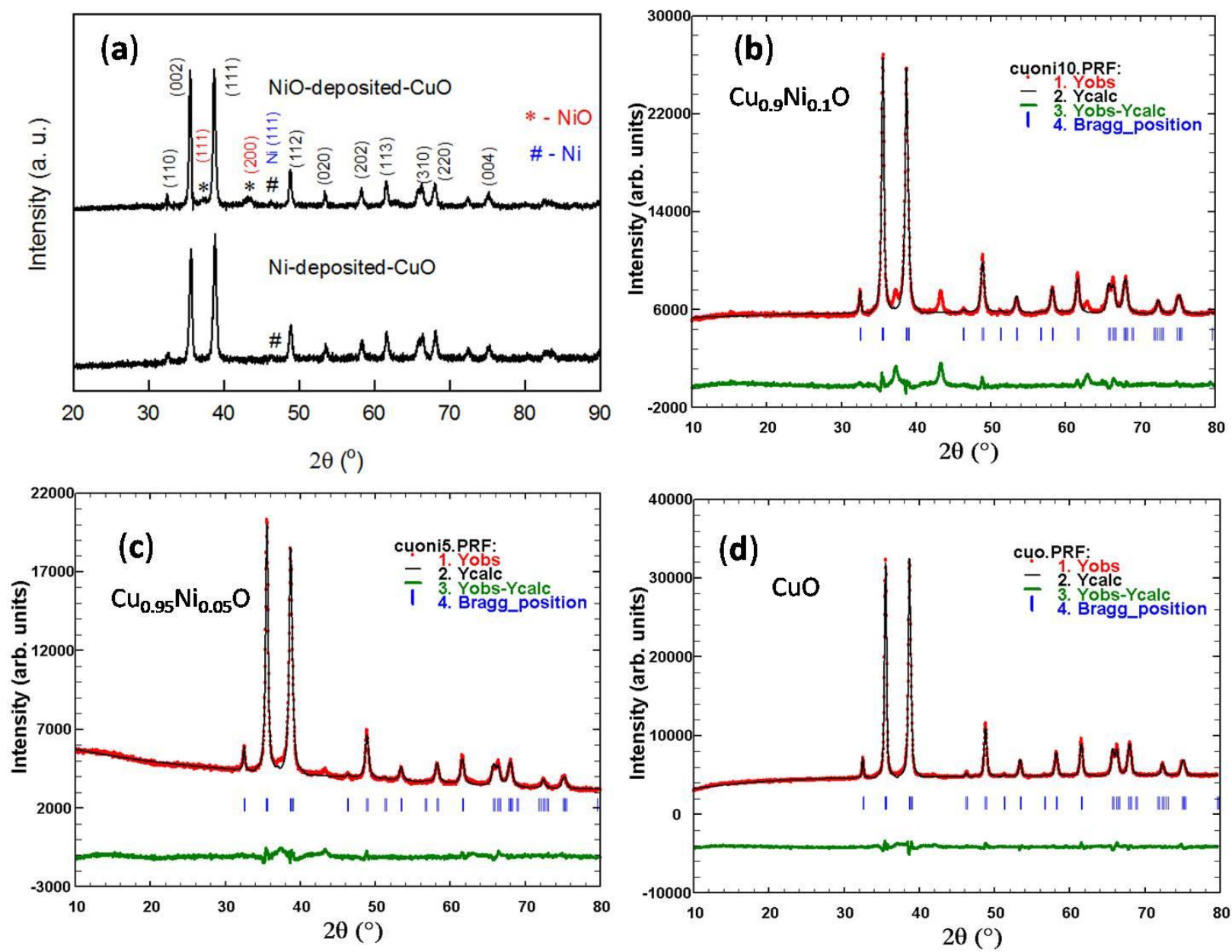

Figure $\mathrm{S} 1$. Comparative $\mathrm{XRD}$ of $\mathrm{NiO}$ and $\mathrm{Ni}$ deposited $\mathrm{CuO}$ catalysts (a). Rietveld refined observed ( $\left.\mathrm{Y}_{\mathrm{obs}}\right)$, calculated ( $\left.\mathrm{Y}_{\text {calc }}\right)$, and difference $\left(\mathrm{Y}_{\mathrm{obs}}-\mathrm{Y}_{\text {calc }}\right)$ XRD patterns of $\mathrm{Cu}_{0.9} \mathrm{Ni}_{0.1} \mathrm{O}(\mathrm{b})$, $\mathrm{Cu}_{0.95} \mathrm{Ni}_{0.05} \mathrm{O}$ (c) and $\mathrm{CuO}(\mathrm{d})$; “"” represents the Bragg position. 


\section{Figure S2}

(a)

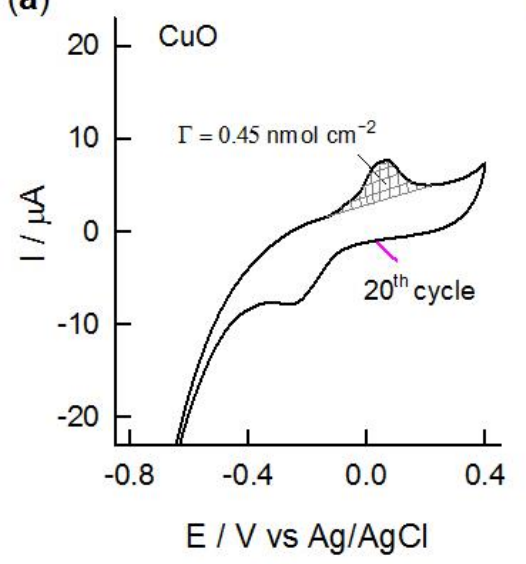

(d)

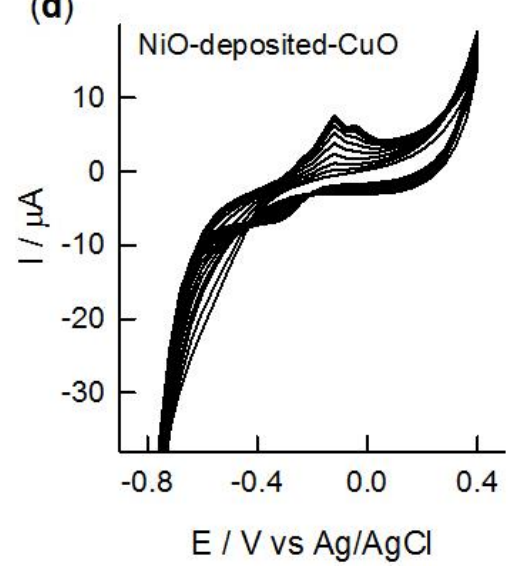

(b)
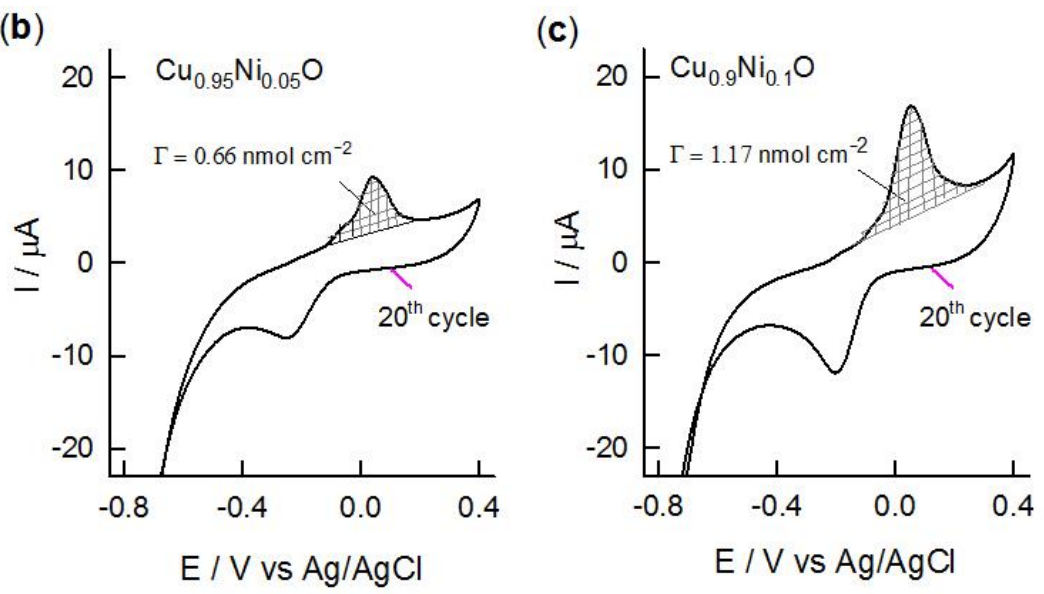

(e)

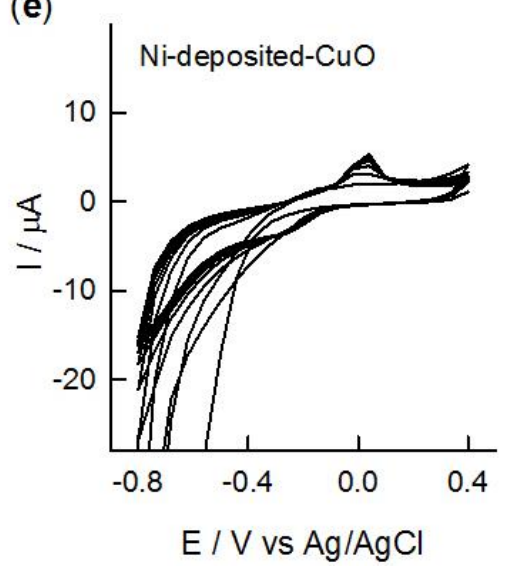

Figure S2. The last CV cycle with their respective calculated surface excess values of $\mathrm{CuO}$ (a) $\mathrm{Cu}_{0.95} \mathrm{Ni}_{0.05} \mathrm{O}$ (b) and $\mathrm{Cu}_{0.9} \mathrm{Ni}_{0.1} \mathrm{O}$ (c), Continuous $\mathrm{CV}$ responses of $\mathrm{NiO}$-deposited-CuO (d) and $\mathrm{Ni}$ metal-deposited-CuO (e) coated GCE at a scan rate of $40 \mathrm{mV} \mathrm{s}^{-1}$ in $0.5 \mathrm{M} \mathrm{NaHCO}$ electrolyte solution. 


\section{Figure S3}
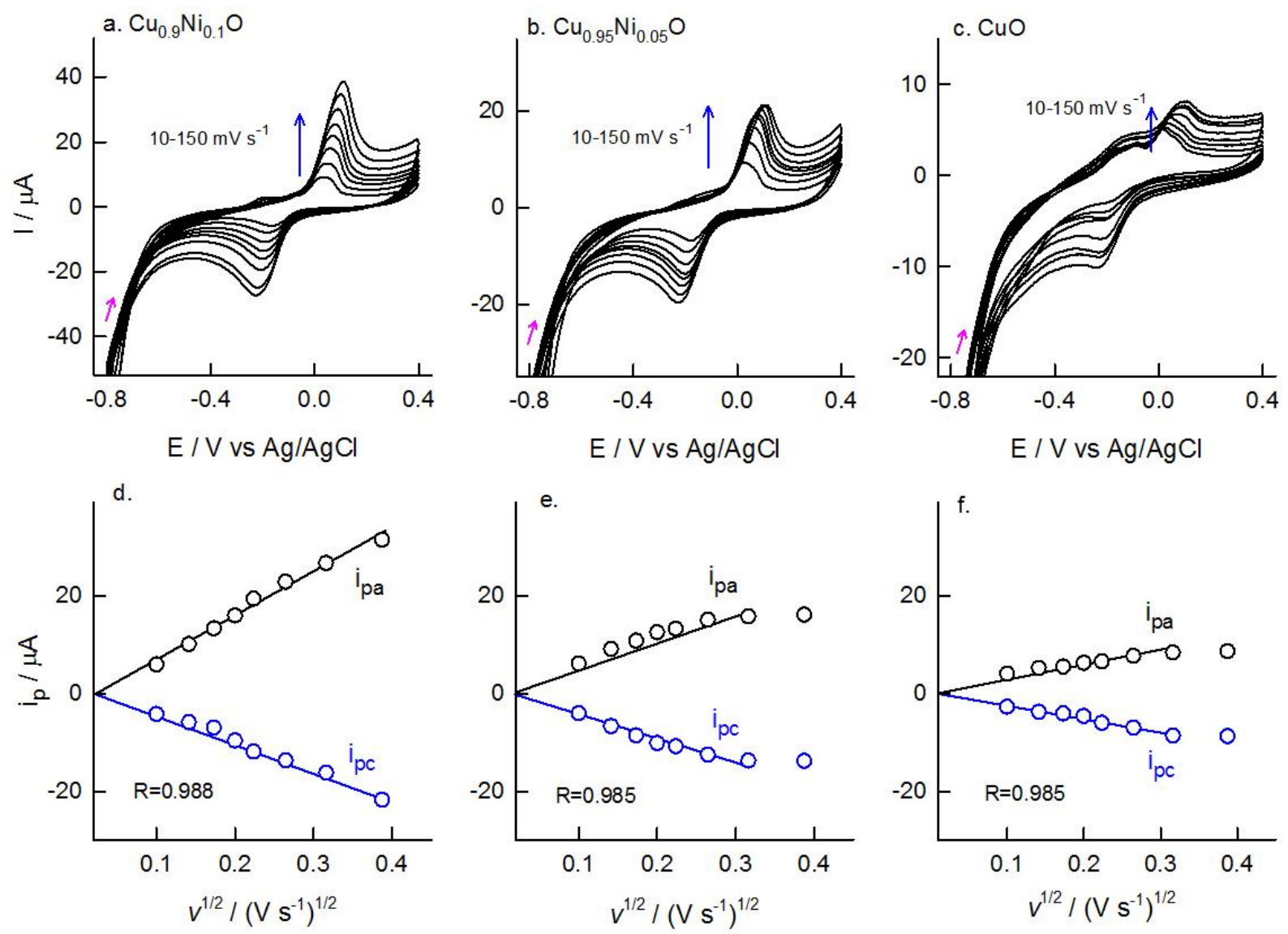

Figure S3. Effect of various $\mathrm{CV}$ scan rates from 10 to $150 \mathrm{mV} \mathrm{s}^{-1}$ on $\mathrm{Cu}_{0.9} \mathrm{Ni}_{0.1} \mathrm{O}$ (a), $\mathrm{Cu}_{0.95} \mathrm{Ni}_{0.05} \mathrm{O}$ (b) and $\mathrm{CuO}$ (c) coated $\mathrm{GCE}$ in $0.5 \mathrm{M} \mathrm{NaHCO}_{3}$ electrolyte solution. Corresponding peak current $\left(\mathrm{i}_{\mathrm{p}}\right)$ versus square root of scan rate $\left(v^{1 / 2}\right)$ plots of $\mathrm{Cu}_{0.9} \mathrm{Ni}_{0.1} \mathrm{O}(\mathrm{d}), \mathrm{Cu}_{0.95} \mathrm{Ni}_{0.05} \mathrm{O}(\mathrm{e})$ and $\mathrm{CuO}(\mathrm{f})$ 
Figure S4
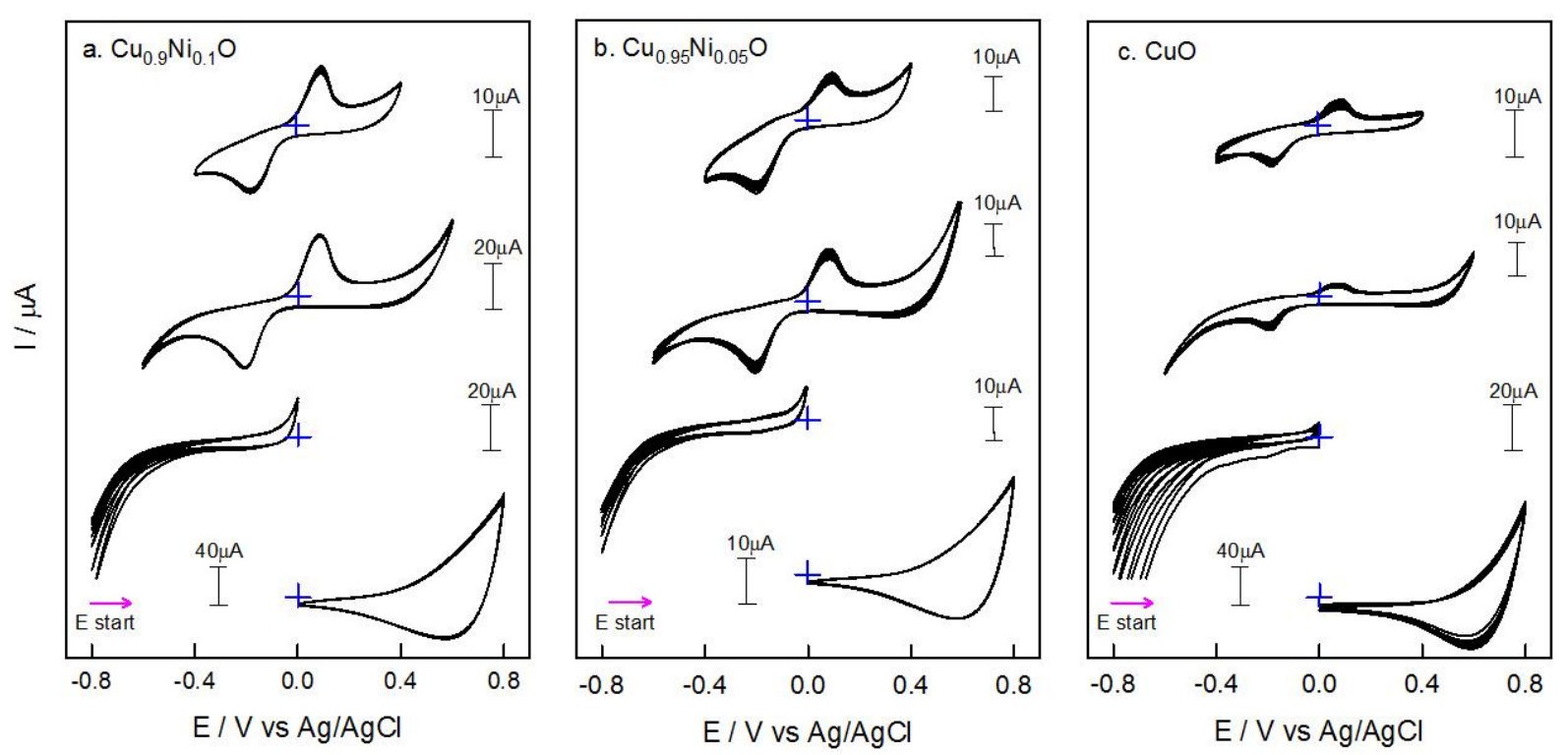

Figure S4. Effect of $\mathrm{CV}$ potential windows on $\mathrm{Cu}_{0.9} \mathrm{Ni}_{0.1} \mathrm{O}$ (a), $\mathrm{Cu}_{0.95} \mathrm{Ni}_{0.05} \mathrm{O}$ (b) and $\mathrm{CuO}$ (c) coated GCE in various ranges from -0.4 to $0.4 \mathrm{~V},-0.6$ to $0.6 \mathrm{~V}, 0$ to $-0.8 \mathrm{~V}$ and 0 to $0.8 \mathrm{~V}$ at a scan rate of $40 \mathrm{mV} \mathrm{s}^{-1}$ in $0.5 \mathrm{M} \mathrm{NaHCO}_{3}$ electrolyte solution. 


\section{Figure S5}

(a)

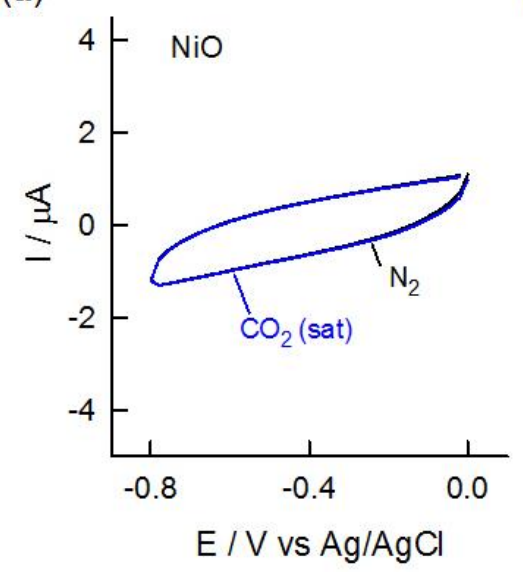

(b)

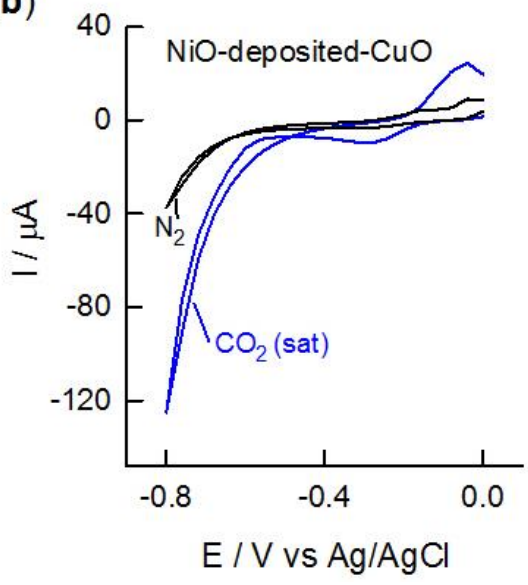

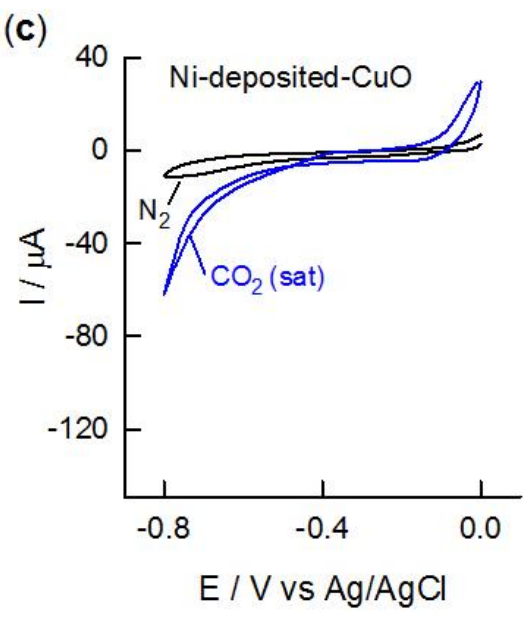

Figure S5. CV response for the electroreduction of $\mathrm{CO}_{2}$ (saturated for 30 mins) on $\mathrm{NiO}$ (a) and $\mathrm{NiO}-$ deposited-CuO (b) and Ni-deposited-CuO (c) coated GCE at a scan rate of $20 \mathrm{mV} \mathrm{s}^{-1}$ in 0.5 $\mathrm{M} \mathrm{NaHCO}_{3}$ electrolyte solution. 
Figure S6

(a)

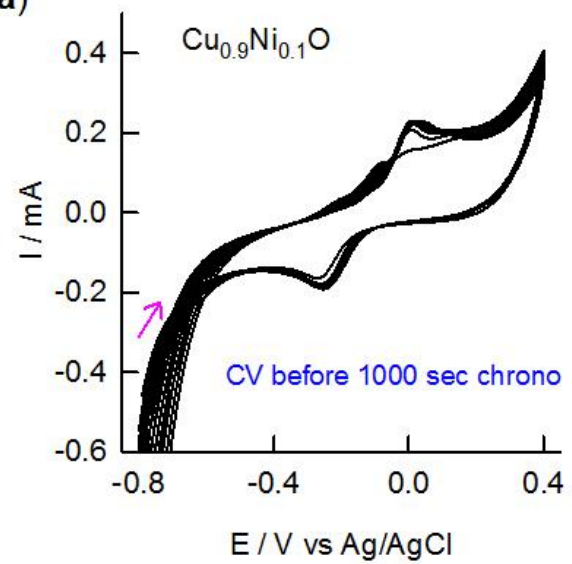

(c)

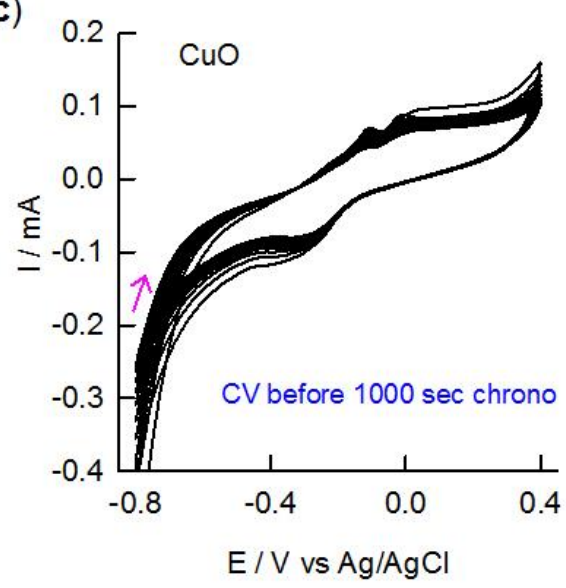

(b)

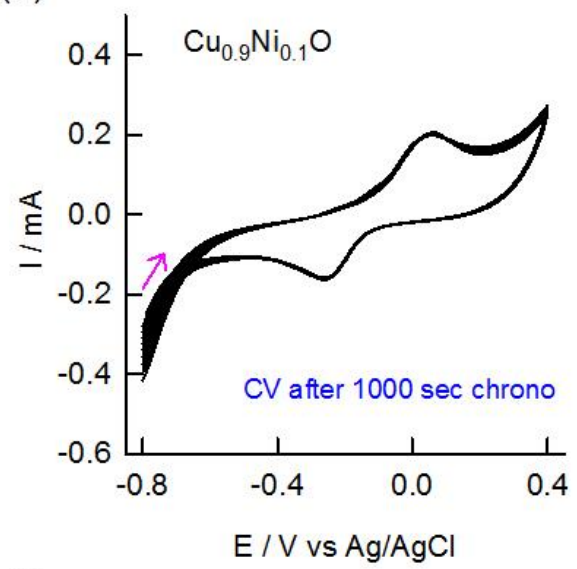

(d)

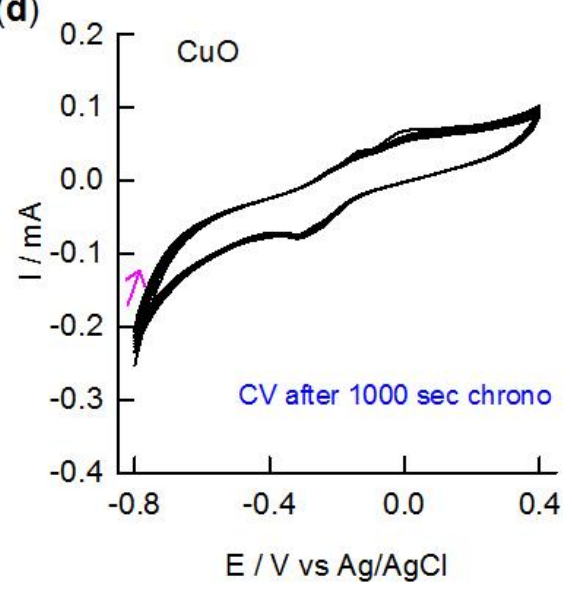

Figure S6. Continuous $\mathrm{CV}$ responses of $\mathrm{Cu}_{0.9} \mathrm{Ni}_{0.1} \mathrm{O}(\mathrm{a}, \mathrm{b})$ and $\mathrm{CuO}(\mathrm{c}, \mathrm{d})$ coated GCE in $0.5 \mathrm{M}$ $\mathrm{NaHCO}_{3}$ electrolyte solution (a, c) and after $1000 \mathrm{sec}$ chronoamperometric experiment at $-0.8 \mathrm{~V}$ in $\mathrm{CO}_{2}$ saturated $0.5 \mathrm{M} \mathrm{NaHCO}_{3}$ electrolyte solution (b, d) at a scan rate of $40 \mathrm{mV} \mathrm{s}^{-1}$. 


\section{Figure S7}

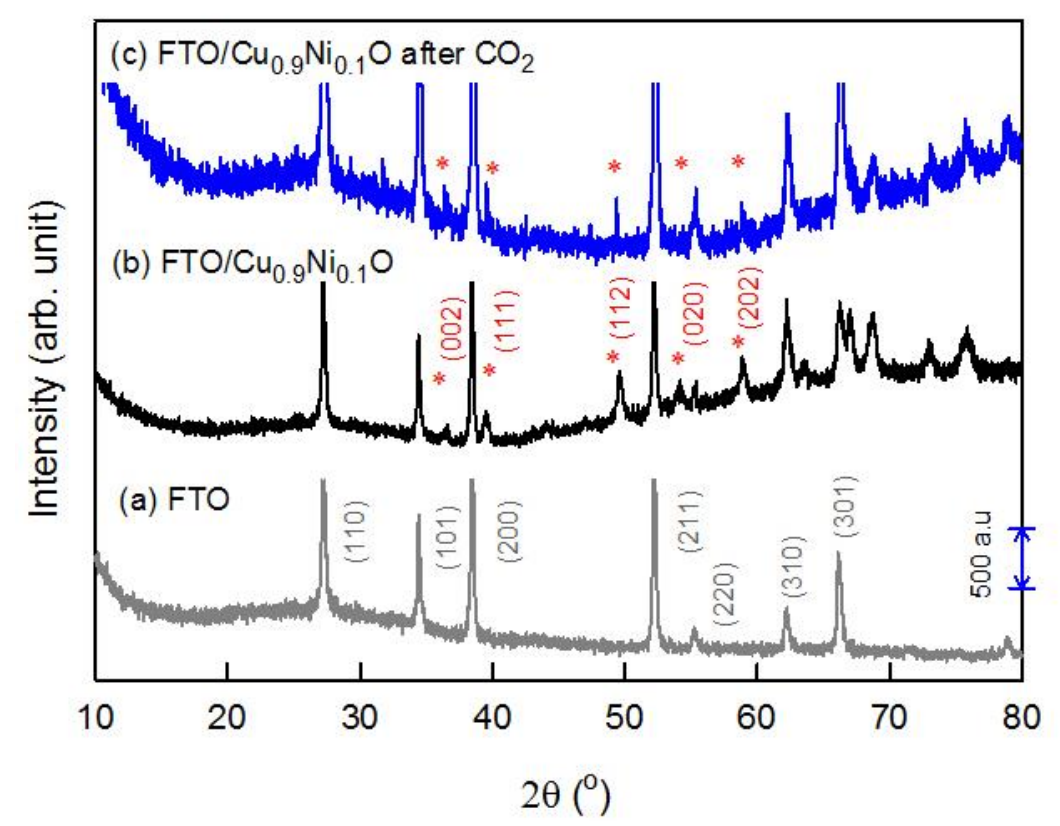

Figure S7. Comparative XRD of bare FTO (a), $\mathrm{Cu}_{0.9} \mathrm{Ni}_{0.1} \mathrm{O}$ coated FTO before (b) and after 1000 sec chronoamperometric $\mathrm{CO}_{2}$ electrocatalysis (c). 


\section{Figure S8}

(a) $\mathrm{Cu}_{0.9} \mathrm{Ni}_{0.1} \mathrm{O}$ after $\mathrm{CO}_{2}$

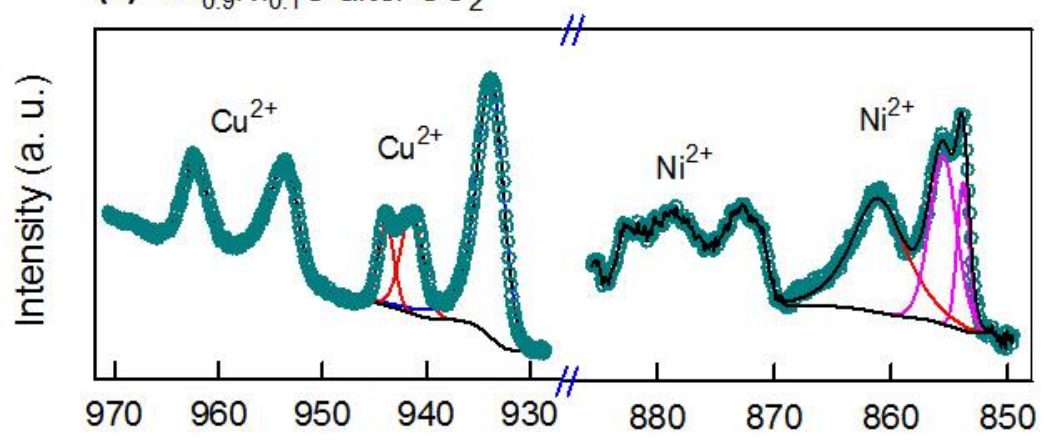

(b) $\mathrm{Cu}_{0.9} \mathrm{Ni}_{0.1} \mathrm{O}$ before $\mathrm{CO}_{2}$

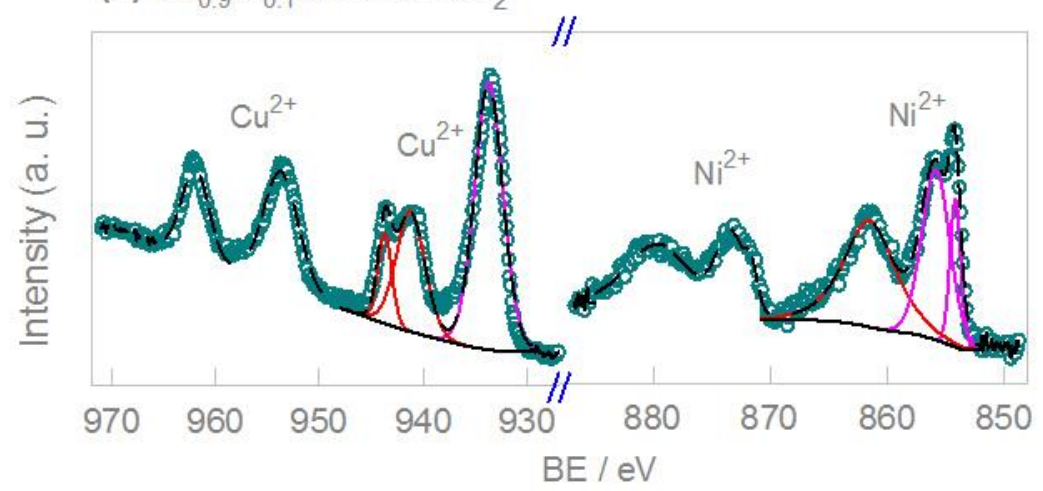

Figure S8. Comparative high resolution XPS $\mathrm{Cu}(2 \mathrm{p})$ and $\mathrm{Ni}(2 \mathrm{p})$ spectra of $\mathrm{Cu}_{0.9} \mathrm{Ni}_{0.1} \mathrm{O}$ after 1000 sec chronoamperometric $\mathrm{CO}_{2}$ electrocatalysis (a) and replicate XPS spectra of as-prepared $\mathrm{Cu}_{0.9} \mathrm{Ni}_{0.1} \mathrm{O}$ catalyst (b). 
Figure S9

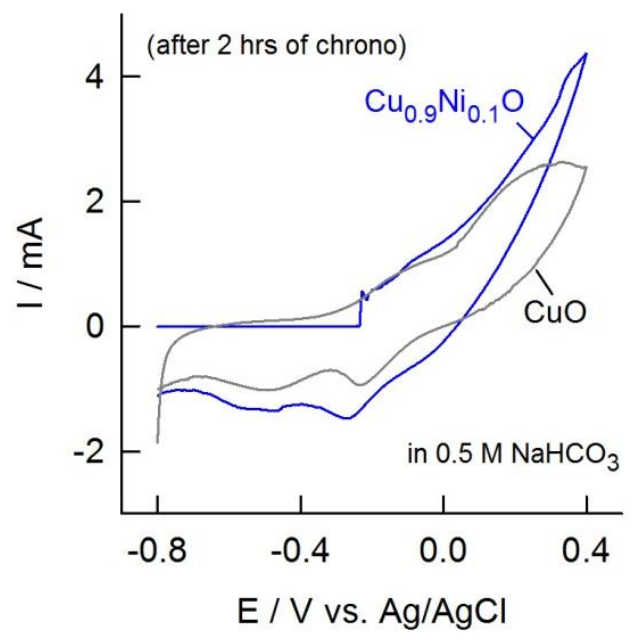

Figure S9. $\mathrm{CV}$ of $\mathrm{CuO}$ and $\mathrm{Cu}_{0.9} \mathrm{Ni}_{0.1} \mathrm{O}$ post chronoamperometry at $-0.8 \mathrm{~V}$ for 2 hours. Behavior depicts the formation of metallic copper. 
Figure S10
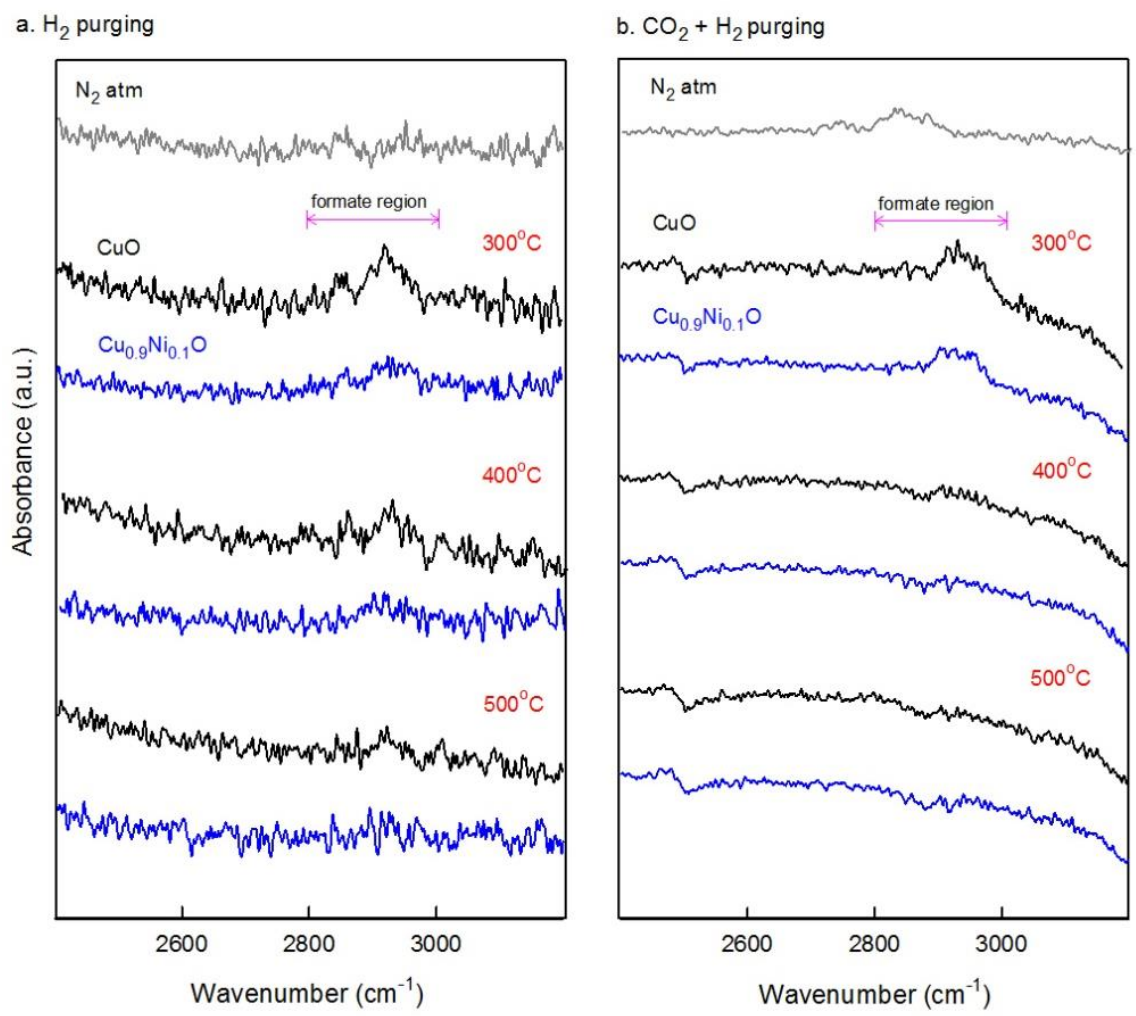

Figure S10. DRIFT spectra recorded in IR ranges (2400 to $3200 \mathrm{~cm}^{-1}$ ) on passing $\mathrm{H}_{2}$ (a) and $\mathrm{CO}_{2}+\mathrm{H}_{2}$ UHP gases (b) over $\mathrm{KBr}$ mixed $\mathrm{Cu}_{0.9} \mathrm{Ni}_{0.1} \mathrm{O}$ and $\mathrm{CuO}$ catalysts at different temperatures from 300 to $500^{\circ} \mathrm{C}$. 
Figure S11

(a)

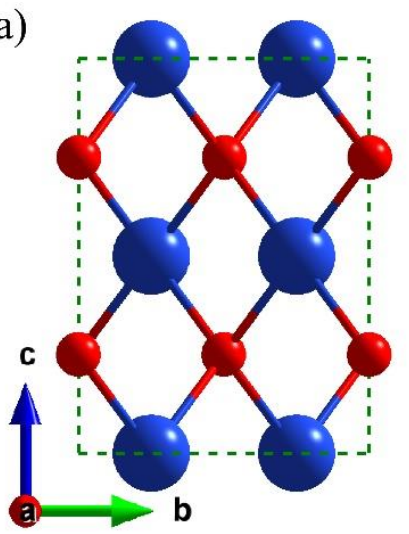

(b)

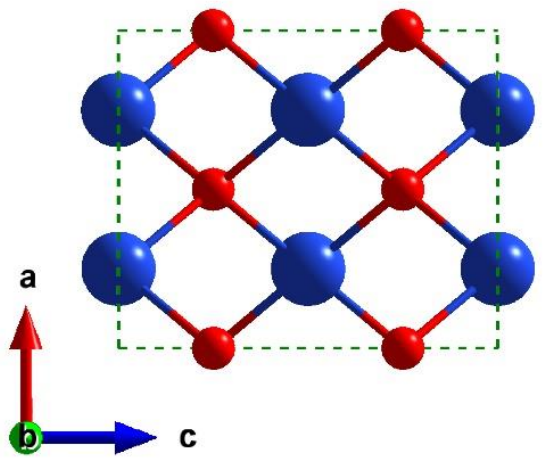

(c)

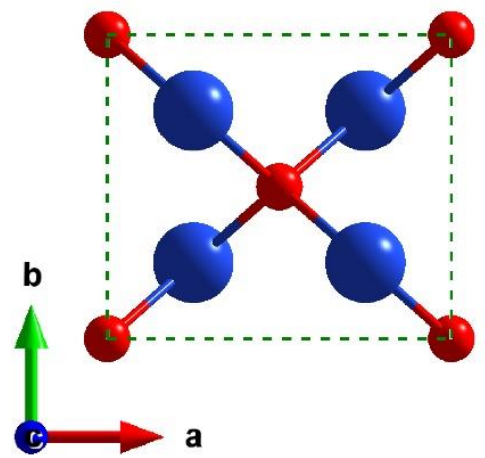

Figure S11. Structures of optimized bulk $\mathrm{CuO}$ in a (a), b (b), and c (c) directions, respectively. The blue and red spheres represent $\mathrm{Cu}$, and $\mathrm{O}$ atoms, respectively.

Figure S12
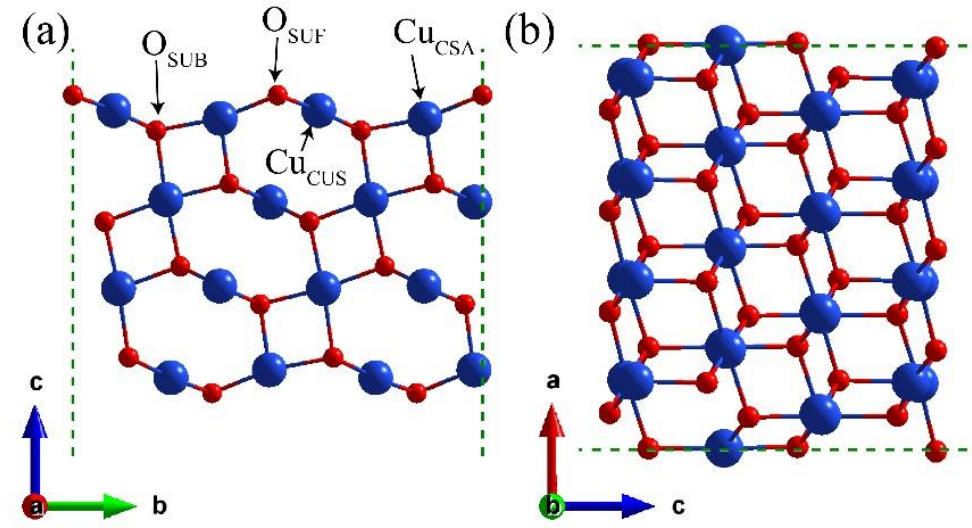

(c)

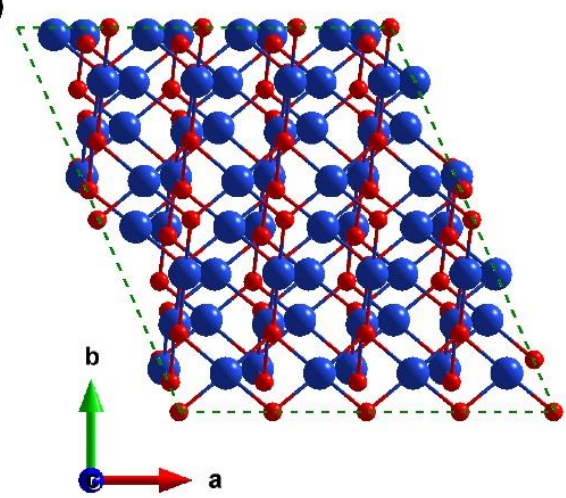

Figure S12. Structures of optimized (111) surface of CuO slab in a (a), b (b), and c (c) directions, respectively. The blue and red spheres represent $\mathrm{Cu}$ and $\mathrm{O}$ atoms, respectively. 
Figure S13

(a)

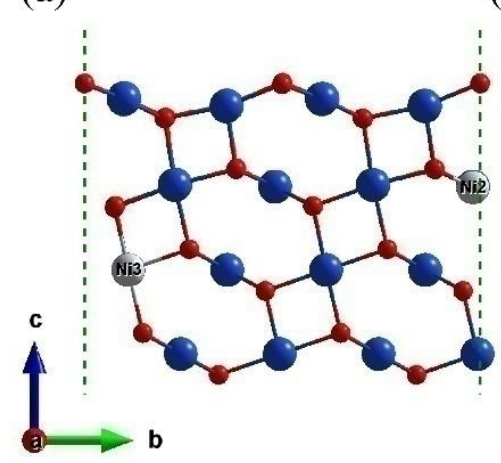

(b)

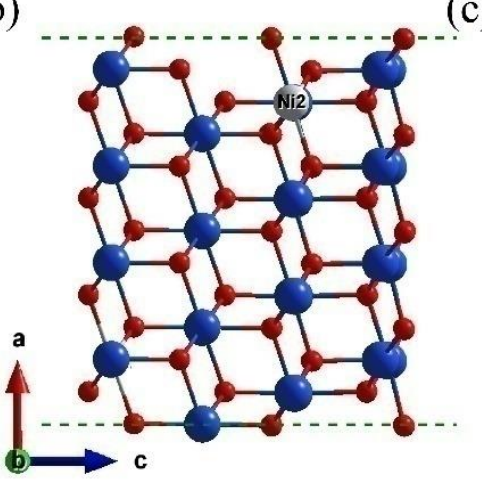

(c)

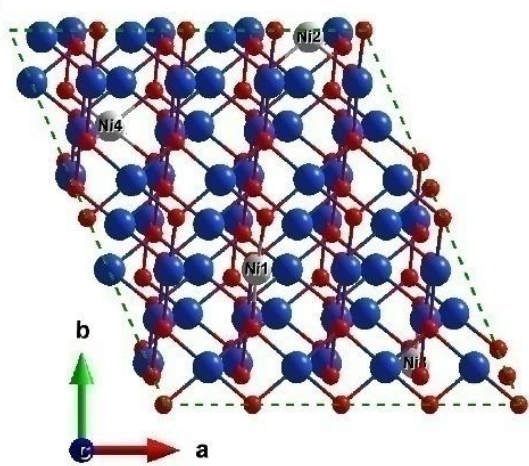

Figure S13. Structures of optimized (111) surface of $\mathrm{Cu}_{0.9375} \mathrm{Ni}_{0.0625} \mathrm{O}$ slab in a (a), b (b), and c (c) directions, respectively. The blue, red, and grey spheres represent $\mathrm{Cu}, \mathrm{O}$, and $\mathrm{Ni}$ atoms, respectively.

The adsorption energies of 7 intermediates involved in the $\mathrm{CO}_{2}$ electro-reduction are calculated according to the following expressions:

$$
\begin{aligned}
& E_{a d s}^{D F T}(\mathrm{COOH})=\mathrm{E}\left(\mathrm{CO}_{2}^{*}\right)-\left[\mathrm{E}^{*}+\mathrm{E}\left(\mathrm{CO}_{2}\right)+0.5 \mathrm{E}\left(\mathrm{H}_{2}\right)\right] \\
& E_{a d s}^{D F T}(\mathrm{CO})=\mathrm{E}\left(\mathrm{CO}^{*}\right)-\left[\mathrm{E}^{*}+\mathrm{E}(\mathrm{CO})\right] \\
& E_{a d s}^{D F T}(\mathrm{CHO})=\mathrm{E}\left(\mathrm{CHO}^{*}\right)-\left[\mathrm{E}^{*}+\mathrm{E}(\mathrm{CO})+0.5 \mathrm{E}\left(\mathrm{H}_{2}\right)\right] \\
& E_{a d s}^{D F T}\left(\mathrm{CH}_{2} \mathrm{O}\right)=\mathrm{E}\left(\mathrm{CH}_{2} \mathrm{O}^{*}\right)-\left[\mathrm{E}^{*}+\mathrm{E}(\mathrm{CO})+\mathrm{E}\left(\mathrm{H}_{2}\right)\right] \\
& E_{a d s}^{D F T}\left(\mathrm{CH}_{3} \mathrm{O}\right)=\mathrm{E}\left(\mathrm{CH}_{3} \mathrm{O}^{*}\right)-\left[\mathrm{E}^{*}+\mathrm{E}(\mathrm{CO})+1.5 \mathrm{E}\left(\mathrm{H}_{2}\right)\right] \\
& E_{a d s}^{D F T}(\mathrm{O})=\mathrm{E}\left(\mathrm{O}^{*}\right)-\left[\mathrm{E}^{*}+0.5 \mathrm{E}\left(\mathrm{O}_{2}\right)\right] \\
& E_{a d s}^{D F T}(\mathrm{OH})=\mathrm{E}\left(\mathrm{O}^{*}\right)-\left[\mathrm{E}^{*}+\mathrm{E}\left(\mathrm{H}_{2} \mathrm{O}\right)-0.5 \mathrm{E}\left(\mathrm{H}_{2}\right)\right]
\end{aligned}
$$


Figure S14

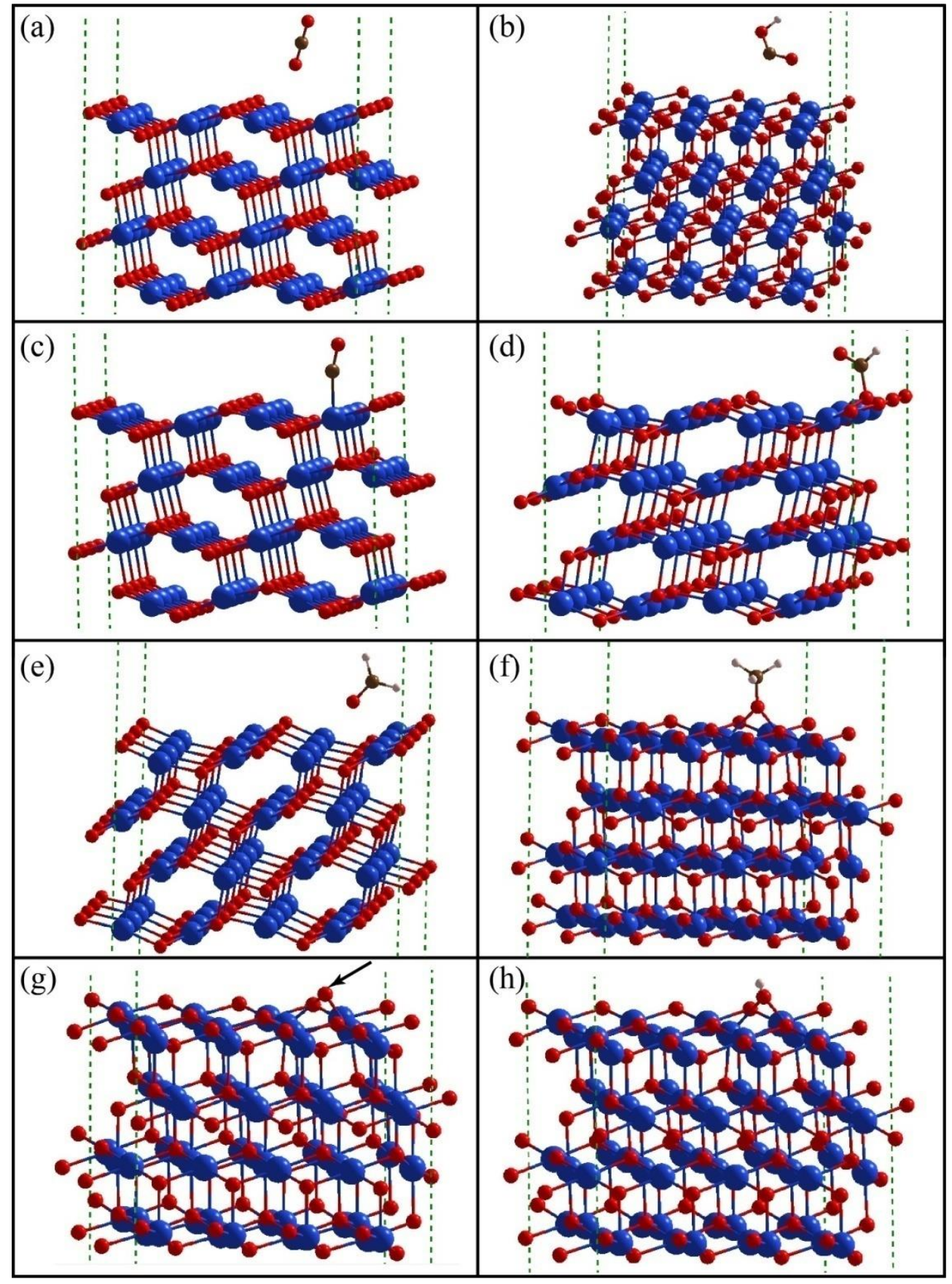

Figure S14. Completely optimized structures of $\mathrm{CO}_{2}$ (a), $\mathrm{COOH}$ (b), $\mathrm{CO}$ (c), $\mathrm{CHO}$ (d), $\mathrm{CH}_{2} \mathrm{O}$ (d), $\mathrm{CH}_{3} \mathrm{O}$ (e), $\mathrm{O}$ (shown by black arrow) (f), and $\mathrm{OH}(\mathrm{d})$ intermediates on (111) surface of $\mathrm{CuO}$. The blue, red, brown, and light pink spheres represent $\mathrm{Cu}, \mathrm{O}, \mathrm{C}$, and $\mathrm{H}$ atoms, respectively. 
Figure S15

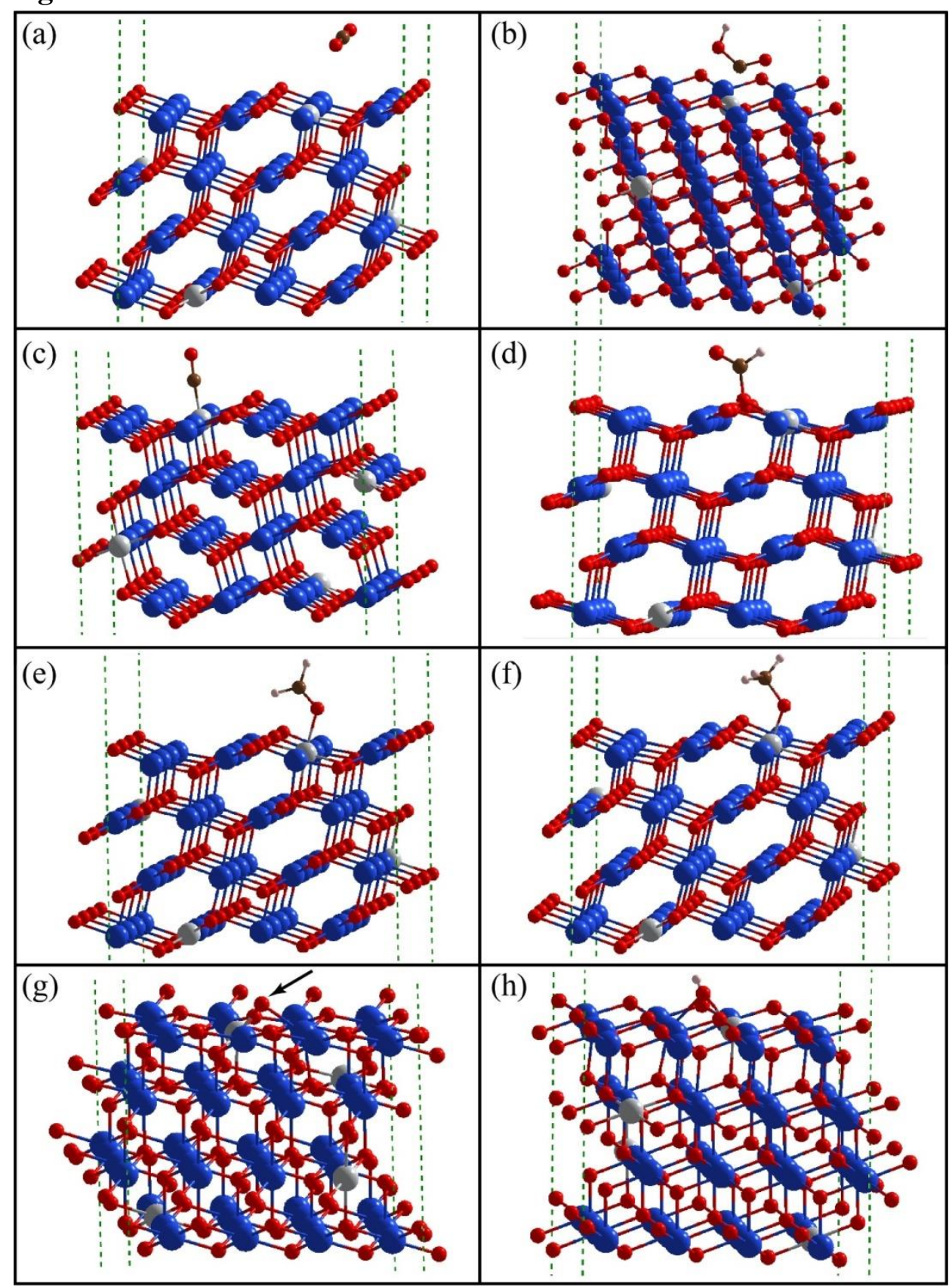

Figure S15. Completely optimized structures of $\mathrm{CO}_{2}$ (a), $\mathrm{COOH}(\mathrm{b}), \mathrm{CO}$ (c), $\mathrm{CHO}$ (d), $\mathrm{CH}_{2} \mathrm{O}$ (d), $\mathrm{CH}_{3} \mathrm{O}$ (e), $\mathrm{O}$ (f), and $\mathrm{OH}$ (d) intermediates on (111) surface of $\mathrm{Cu}_{0.9375} \mathrm{Ni}_{0.0625} \mathrm{O}$. The blue, red, grey, brown, and light pink spheres represent $\mathrm{Cu}, \mathrm{O}, \mathrm{Ni}, \mathrm{C}$, and $\mathrm{H}$ atoms, respectively. 


\section{Figure S16}

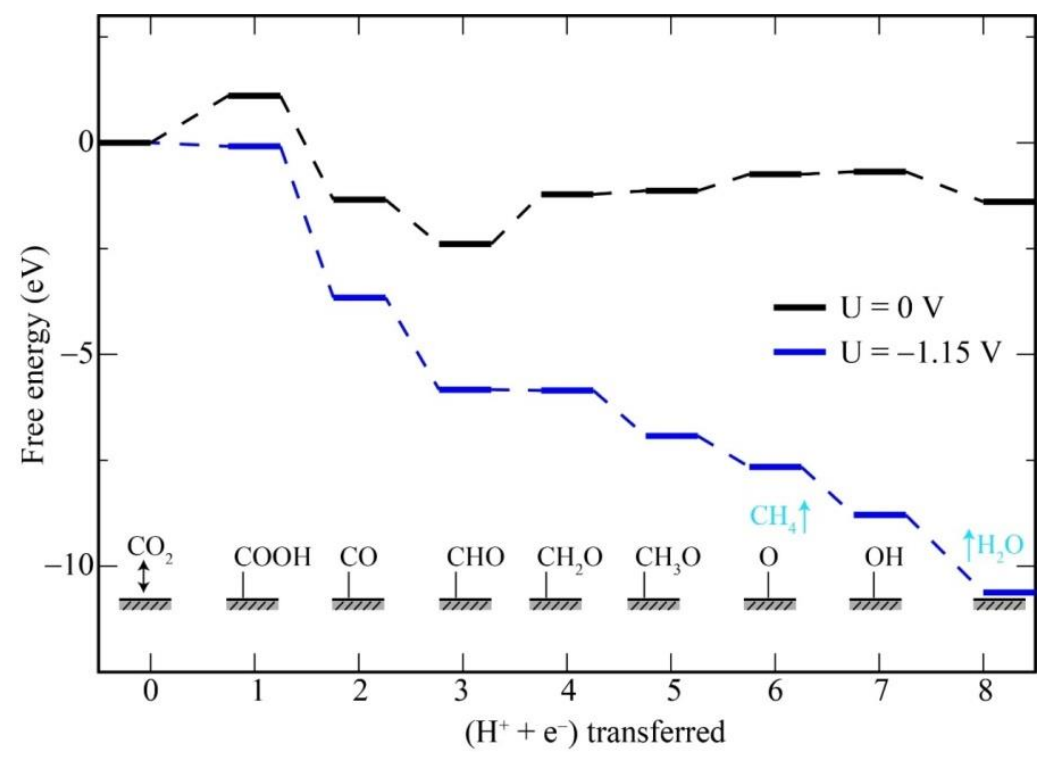

Figure S16. Free energy diagram for electroreduction of $\mathrm{CO}_{2}$ to $\mathrm{CH}_{4}$ on (111) surface of $\mathrm{CuO}$. 
Table S1. Rietveld refined structural parameters for $\mathrm{CuO}, \mathrm{Cu}_{0.95} \mathrm{Ni}_{0.05} \mathrm{O}$ and $\mathrm{Cu}_{0.90} \mathrm{Ni}_{0.10} \mathrm{O}$ compounds.

\begin{tabular}{|c|c|c|c|}
\hline Compounds & $\mathrm{CuO}$ & $\mathrm{Cu}_{0.95 \mathrm{Ni}}{ }_{0.05} \mathrm{O}$ & Cuo.90Nio.10O \\
\hline Crystal System & Monoclinic & Monoclinic & Monoclinic \\
\hline Space group & C $12 /$ c 1 (No. 15) & C $12 /$ c 1 (No. 15) & C $12 /$ c 1 (No. 15) \\
\hline \multicolumn{4}{|l|}{ Lattice Parameters $(\AA)$} \\
\hline$a$ & $4.687(7)$ & $4.682(8)$ & $4.683(7)$ \\
\hline$b$ & $3.431(4)$ & $3.429(5)$ & $3.429(9)$ \\
\hline$c$ & $5.136(7)$ & $5.131(9)$ & $5.132(8)$ \\
\hline Cell volume $\left(\AA^{3}\right)$ & $81.52(2)$ & $81.30(3)$ & $81.33(4)$ \\
\hline$\beta\left(^{\circ}\right)$ & $99.36(4)$ & $99.28(7)$ & $99.27(5)$ \\
\hline \multicolumn{4}{|l|}{$R_{\text {Factors }}$} \\
\hline$R_{\text {Bragg }}$ & 2.52 & 6.22 & 8.05 \\
\hline$R_{\mathrm{F}}$ & 2.55 & 6.82 & 8.01 \\
\hline
\end{tabular}

Table S2. Comparative electrocatalytic performance of the catalyst: Current values are taken from CVs towards for $\mathrm{CO}_{2}$ electroreduction reaction. BET surface area is used for normalization.

\begin{tabular}{ccc}
\hline Catalyst & Current $(\mathbf{m A})$ & Current density $\left(\mathbf{m A ~ c m} \mathbf{~ c m}^{-2}\right)$ \\
\hline $\mathrm{CuO}$ & 0.10 & 0.063 \\
$\mathrm{Cu}_{0.95} \mathrm{Ni}_{0.05} \mathrm{O}$ & 0.18 & 0.360 \\
$\mathrm{Cu}_{0.9} \mathrm{Ni}_{0.1} \mathrm{O}$ & 0.29 & 0.400 \\
$\mathrm{Ni} / \mathrm{CuO}$ & 0.06 & 0.012 \\
$\mathrm{NiO} / \mathrm{CuO}$ & 0.13 & 0.026 \\
\hline
\end{tabular}


Table S3. Comparative electrocatalytic performance of $\mathrm{Cu}_{0.90} \mathrm{Ni}_{0.10} \mathrm{O}$ catalyst with other copper based electrocatalysts

\begin{tabular}{|c|c|c|c|c|c|}
\hline S.No & Electrocatalyst & Electrolyte & $\begin{array}{c}\text { Applied } \\
\text { potentials }\end{array}$ & Products (FE\%) & Total \\
\hline 1 & Polycrystalline $\mathrm{Cu}^{1}$ & $0.1 \mathrm{M} \mathrm{KHCO}_{3}$ & $-1.05 \mathrm{~V}_{\mathrm{RHE}}$ & $\begin{array}{l}\mathrm{CH}_{4}(24.4) ; \mathrm{C}_{2} \mathrm{H}_{4}(26.0), \mathrm{CO} \\
(1.1) \mathrm{H}_{2}(23.0), \mathrm{C}_{2} \mathrm{H}_{5} \mathrm{OH} \\
(9.8), \mathrm{HCOOH}(2.1)\end{array}$ & $91.2 \%$ \\
\hline 2 & $\mathrm{Cu}$ nanoparticles ${ }^{2}$ & $0.1 \mathrm{M} \mathrm{KClO}_{4}$ & $-1.1 \mathrm{~V}_{\mathrm{RHE}}$ & $\mathrm{CH}_{4}(1), \mathrm{C}_{2} \mathrm{H}_{4}(36), \mathrm{CO}(34)$ & $72.0 \%$ \\
\hline 3 & $\mathrm{Cu}$ nano particles ${ }^{3}$ & $0.1 \mathrm{M} \mathrm{NaHCO}_{3}$ & $-1.25 \mathrm{~V}_{\mathrm{RHE}}$ & $\mathrm{CH}_{4}(80 \%) ; \mathrm{H}_{2}(13 \%)$ & $93.0 \%$ \\
\hline 4 & $\mathrm{Cu}$ nanoflower ${ }^{4}$ & $0.1 \mathrm{M} \mathrm{KHCO}_{3}$ & $-1.6 V_{\mathrm{RHE}}$ & $\begin{array}{l}\mathrm{CH}_{4}(5) ; \mathrm{C}_{2} \mathrm{H}_{4}(10) ; \mathrm{H}_{2}(29) ; \\
\mathrm{HCOOH}(49)\end{array}$ & $94.0 \%$ \\
\hline 5 & $\mathrm{Au}_{3} \mathrm{Cu}^{5}$ & $0.1 \mathrm{M} \mathrm{KHCO}_{3}$ & $-0.73 \mathrm{~V}_{\mathrm{RHE}}$ & $\mathrm{CO}$ (64.7), $\mathrm{HCOOH}(3.1)$ & $67.8 \%$ \\
\hline 6 & $\mathrm{CuIn}^{6}$ & $0.1 \mathrm{M} \mathrm{KHCO}_{3}$ & $-0.6 \mathrm{~V}_{\mathrm{RHE}}$ & $\mathrm{CO}(38), \mathrm{HCOOH}(34)$ & $72.0 \%$ \\
\hline 7 & Oxide derived $\mathrm{Cu}^{7}$ & $0.5 \mathrm{M} \mathrm{NaHCO}_{3}$ & $-0.5 \mathrm{~V}_{\mathrm{RHE}}$ & $\mathrm{CO}(40), \mathrm{HCOOH}(33)$ & $73.0 \%$ \\
\hline 8 & $\begin{array}{l}\text { Oxide derived } \mathrm{Cu} \\
\text { foam }^{8}\end{array}$ & $0.5 \mathrm{M} \mathrm{NaHCO}_{3}$ & $-1.0 \mathrm{~V}_{\mathrm{RHE}}$ & $\begin{array}{l}\mathrm{CO}(5), \mathrm{HCOOH}(5), \mathrm{C}_{2} \mathrm{H}_{4} \\
(20) ; \mathrm{C}_{2} \mathrm{H}_{6}(25)\end{array}$ & $55.0 \%$ \\
\hline 9 & $\mathrm{Cu} / \mathrm{SnO}_{2}{ }^{9}$ & $0.5 \mathrm{M} \mathrm{KHCO}_{3}$ & $-0.7 V_{\mathrm{RHE}}$ & $\mathrm{CO}(93)$ & $93.0 \%$ \\
\hline 10 & $\mathrm{Cu}_{2} \mathrm{O}^{10}$ & $0.5 \mathrm{M} \mathrm{KHCO}_{3}$ & $-1.82 \mathrm{~V}_{\mathrm{Ag} / \mathrm{AgCl}}$ & $\begin{array}{l}\mathrm{CO}(6), \mathrm{CH}_{4}(1), \mathrm{C}_{2} \mathrm{H}_{4}(26) \\
\mathrm{HCOOH}(8)\end{array}$ & $41.0 \%$ \\
\hline 11 & $\mathrm{Cu}_{2} \mathrm{O}^{11}$ & $0.1 \mathrm{M} \mathrm{KHCO}_{3}$ & $-1.1 \mathrm{~V}_{\mathrm{RHE}}$ & $\begin{array}{l}\mathrm{CO}(3), \mathrm{CH}_{4}(4), \mathrm{C}_{2} \mathrm{H}_{4}(33) \\
\mathrm{C}_{2} \mathrm{H}_{6}(9), \mathrm{HCOOH}(22)\end{array}$ & $71.0 \%$ \\
\hline 12 & $\mathrm{CuO}$ nanoparticles ${ }^{12}$ & $0.2 \mathrm{M} \mathrm{KHCO}_{3}$ & $-1.7 \mathrm{~V}_{\mathrm{SCE}}$ & $\begin{array}{l}\mathrm{C}_{2} \mathrm{H}_{5} \mathrm{OH}(15.5), \text { n-propanol } \\
(3.6)\end{array}$ & $19.1 \%$ \\
\hline \multirow{3}{*}{13} & \multirow{3}{*}{$\mathrm{Cu}_{0.9} \mathrm{Ni}_{0.1} \mathrm{O}$} & $0.2 \mathrm{M} \mathrm{KI}$ & & $\begin{array}{l}\mathrm{C}_{2} \mathrm{H}_{5} \mathrm{OH}(36.1), \text { n-propanol } \\
\text { (3) }\end{array}$ & $39.1 \%$ \\
\hline & & \multirow[t]{2}{*}{$0.5 \mathrm{M} \mathrm{NaHCO}_{3}$} & \multirow[t]{2}{*}{$\begin{array}{c}\mathbf{- 0 . 8} \mathbf{V}_{\mathrm{Ag} / \mathrm{AgCl}} \\
\left(-0.2 \mathrm{~V}_{\mathrm{RHE}}\right)\end{array}$} & $\begin{array}{l}\mathrm{CO}(<1), \mathrm{CH}_{4}(29.4), \mathrm{C}_{2} \mathrm{H}_{4} \\
(12.9), \mathrm{H}_{2}(25.4)\end{array}$ & $83.0 \%$ \\
\hline & & & & $\mathrm{C}_{2} \mathrm{H}_{5} \mathrm{OH}(15.3)$ & \\
\hline
\end{tabular}

RHE-reversible hydrogen electrode; SCE-standard calomel electrode; $\mathrm{Ag} / \mathrm{AgCl}$-Silver/Silver chloride electrode 
Table S4. DFT energies ( $\left.\mathrm{E}^{\mathrm{DFT}}\right)$, corrections to free energy $(\mathrm{ZPE}-\mathrm{TS})^{13}$, and free energies of adsorption $(\mathrm{G})$ of isolated adsorbates which are used as reference for calculating reaction free energies

\begin{tabular}{lccc}
\hline Species & $\mathbf{E}^{\mathbf{D F T}}(\mathbf{e V})$ & $\mathbf{Z P E}-\mathbf{T S}^{\mathbf{1 3}}(\mathbf{e V})$ & $\mathbf{G}(\mathbf{e V})$ \\
\hline $\mathrm{CO}_{2}$ & -22.95 & -0.34 & -23.29 \\
$\mathrm{CO}$ & -14.77 & -0.53 & -15.30 \\
$\mathrm{H}_{2} \mathrm{O}$ & -14.22 & -0.07 & -14.29 \\
$\mathrm{H}_{2}$ & -6.77 & -0.15 & -6.92 \\
$\mathrm{O}_{2}$ & -9.86 & -0.19 & -10.05 \\
\hline
\end{tabular}

Table S5. DFT adsorption energies $\left(E_{\text {ads }}^{D F T}\right)$, corrections to free energy (ZPE - TS), and free energies of adsorption $\left(\mathrm{G}^{*}\right)$ of intermediates on (111) surface of $\mathrm{CuO}$

\begin{tabular}{lccc}
\hline Intermediates & $\boldsymbol{E}_{\boldsymbol{a d s}}^{\boldsymbol{D F T}}(\mathbf{e V})$ & $\mathbf{Z P E}-\mathbf{T S}(\mathbf{e V})$ & $\mathbf{G}^{*}(\mathbf{e V})$ \\
\hline$* \mathrm{COOH}$ & 0.35 & 0.31 & 1.07 \\
$* \mathrm{CO}$ & -1.85 & -0.03 & -1.35 \\
$* \mathrm{CHO}$ & -3.35 & 0.38 & -2.36 \\
$* \mathrm{CH}_{2} \mathrm{O}$ & -2.34 & 0.46 & -1.20 \\
$* \mathrm{CH}_{3} \mathrm{O}$ & -2.82 & 0.92 & -1.15 \\
$* \mathrm{O}$ & -0.83 & 0.02 & -0.72 \\
$* \mathrm{OH}$ & -0.99 & 0.31 & -0.69 \\
\hline
\end{tabular}


Table S6. DFT adsorption energies $\left(E_{a d s}^{D F T}\right)$, corrections to free energy (ZPE - TS), and free energies of intermediates $\left(\mathrm{G}^{*}\right)$ on $(111)$ surface of $\mathrm{Cu}_{0.9375} \mathrm{Ni}_{0.0625} \mathrm{O}$

\begin{tabular}{lccc}
\hline Intermediates & $\boldsymbol{E}_{\boldsymbol{a d} \boldsymbol{s} \boldsymbol{s}}^{\boldsymbol{D F T}}(\mathbf{e V})$ & ZPE - TS(eV) & G* (eV) \\
\hline$* \mathrm{COOH}$ & -1.06 & 0.45 & -0.20 \\
$* \mathrm{CO}$ & -0.85 & 0.06 & -0.26 \\
$* \mathrm{CHO}$ & -3.12 & 0.36 & -2.15 \\
$* \mathrm{CH}_{2} \mathrm{O}$ & -2.64 & 0.63 & -1.33 \\
$* \mathrm{CH}_{3} \mathrm{O}$ & -2.77 & 0.93 & -1.09 \\
$* \mathrm{O}$ & -0.63 & 0.02 & -0.52 \\
$* \mathrm{OH}$ & -0.71 & 0.30 & -0.42 \\
\hline
\end{tabular}

\section{REFERENCES (supp info)}

(1) Kuhl, K. P.; Cave, E. R.; Abram, D. N.; Jaramillo, T. F. New Insights into the Electrochemical Reduction of Carbon Dioxide on Metallic Copper Surfaces. Energy Environ. Sci. 2012, 5 (5), 7050-7059.

(2) Tang, W.; Peterson, A. A.; Varela, A. S.; Jovanov, Z. P.; Bech, L.; Durand, W. J.; Dahl, S.; Norskov, J. K.; Chorkendorff, I. The Importance of Surface Morphology in Controlling the Selectivity of Polycrystalline Copper for $\mathrm{CO}_{2}$ Electroreduction. Phys. Chem. Chem. Phys. 2012, 14 (1), 76-81.

(3) Manthiram, K.; Beberwyck, B. J.; Alivisatos, A. P. Enhanced Electrochemical Methanation of Carbon Dioxide with a Dispersible Nanoscale Copper Catalyst. J. Am. Chem. Soc. 2014, 136 (38), 13319-13325.

(4) Xie, J.-F.; Huang, Y.-X.; Li, W.-W.; Song, X.-N.; Xiong, L.; Yu, H.-Q. Efficient Electrochemical $\mathrm{CO}_{2}$ Reduction on a Unique Chrysanthemum-like $\mathrm{Cu}$ Nanoflower Electrode and Direct Observation of Carbon Deposite. Electrochim. Acta 2014, 139, 137144. 
(5) Kim, D.; Resasco, J.; Yu, Y.; Asiri, A. M.; Yang, P. Synergistic Geometric and Electronic Effects for Electrochemical Reduction of Carbon Dioxide Using Gold-Copper Bimetallic Nanoparticles. Nature Communi. 2014, 5, 4948-4956.

(6) Rasul, S.; Anjum, D. H.; Jedidi, A.; Minenkov, Y.; Cavallo, L.; Takanabe, K. A Highly Selective Copper-Indium Bimetallic Electrocatalyst for the Electrochemical Reduction of Aqueous $\mathrm{CO}_{2}$ to CO. Angew. Chem. Int. Ed. Engl. 2015, 54 (7), 2146-2150.

(7) Li, C. W.; Kanan, M. W. $\mathrm{CO}_{2}$ Reduction at Low Overpotential on Cu Electrodes Resulting from the Reduction of Thick $\mathrm{Cu}_{2} \mathrm{O}$ Films. J. Am. Chem. Soc. 2012, 134 (17), 7231-7234.

(8) Dutta, A.; Rahaman, M.; Luedi, N. C.; Mohos, M.; Broekmann, P. Morphology Matters: Tuning the Product Distribution of $\mathrm{CO}_{2}$ Electroreduction on Oxide-Derived $\mathrm{Cu}$ Foam Catalysts. ACS Catal. 2016, 6 (6), 3804-3814.

(9) Li, Q.; Fu, J.; Zhu, W.; Chen, Z.; Shen, B.; Wu, L.; Xi, Z.; Wang, T.; Lu, G.; Zhu, J. J.; Sun, S. Tuning Sn-Catalysis for Electrochemical Reduction of $\mathrm{CO}_{2}$ to $\mathrm{CO}$ via the Core/Shell Cu/SnO 2 Structure. J. Am. Chem. Soc. 2017, 139 (12), 4290-4293.

(10) Kim., D.; Lee., S.; Ocon., J. D.; Jeong., B.; Leeb., J. K.; Lee., J. Insights into an Autonomously Formed Oxygen-evacuated $\mathrm{Cu}_{2} \mathrm{O}$ Electrode for the Selective Production of $\mathrm{C}_{2} \mathrm{H}_{4}$ from $\mathrm{CO}_{2}$. Phys. Chem. Chem. Phys. 2015, 17, 824-830.

(11) Kas, R.; Kortlever, R.; Milbrat, A.; Koper, M. T.; Mul, G.; Baltrusaitis, J. Electrochemical $\mathrm{CO}_{2}$ Reduction on $\mathrm{Cu}_{2} \mathrm{O}$-derived Copper Nanoparticles: Controlling the Catalytic Selectivity of Hydrocarbons. Phys. Chem. Chem. Phys. 2014, 16 (24), 12194-12201.

(12) Chi., D.; Yang., H.; Du., Y.; Lv., T.; Sui., G.; Wang., H.; Lu, J. Morphology-Controlled $\mathrm{CuO}$ Nanoparticles for Electroreduction of $\mathrm{CO}_{2}$ to Ethanol. RSC Adv. 2014, 4, 3732937332.

(13) Peterson, A. A.; Abild-Pedersen, F.; Studt, F.; Rossmeisl, J.; Norskov, J.K.; How Copper Catalyzes the Electroreduction of Carbon Dioxide into Hydrocarbon Fuels. Energy Environ. Sci. 2010, 3, 1311-1315. 